University of Nebraska - Lincoln

DigitalCommons@University of Nebraska - Lincoln

Biomedical Imaging and Biosignal Analysis

Laboratory

Biological Systems Engineering

$12-2015$

\title{
A New Method for Shear Wave Speed Estimation in Shear Wave Elastography
}

\author{
Aaron J. Engel \\ University of Nebraska - Lincoln \\ Gregory R. Bashford \\ University of Nebraska - Lincoln, gbashford2@unl.edu
}

Follow this and additional works at: https://digitalcommons.unl.edu/biba

Part of the Biochemistry, Biophysics, and Structural Biology Commons, Bioinformatics Commons, Health Information Technology Commons, Other Analytical, Diagnostic and Therapeutic Techniques and Equipment Commons, and the Systems and Integrative Physiology Commons

Engel, Aaron J. and Bashford, Gregory R., "A New Method for Shear Wave Speed Estimation in Shear Wave Elastography" (2015). Biomedical Imaging and Biosignal Analysis Laboratory. 24.

https://digitalcommons.unl.edu/biba/24

This Article is brought to you for free and open access by the Biological Systems Engineering at DigitalCommons@University of Nebraska - Lincoln. It has been accepted for inclusion in Biomedical Imaging and Biosignal Analysis Laboratory by an authorized administrator of DigitalCommons@University of Nebraska - Lincoln. 


\title{
A New Method for Shear Wave Speed Estimation in Shear Wave Elastography
}

\author{
Aaron J. Engel, Student Member, IEEE, and Gregory R. Bashford, Senior Member, IEEE
}

\begin{abstract}
Visualization of mechanical properties of tissue can aid in noninvasive pathology diagnosis. Shear wave elastography (SWE) measures the elastic properties of soft tissues by estimation of local shear wave propagation speed. In this paper, a new robust method for estimation of shear wave speed is introduced which has the potential for simplifying continuous filtering and real-time elasticity processing. Shear waves were generated by external mechanical excitation and imaged at a high frame rate. Three homogeneous phantoms of varying elastic moduli and one inclusion phantom were imaged. Waves propagating in separate directions were filtered and shear wave speed was estimated by inversion of the 1-D first-order wave equation. Final 2-D shear wave speed maps were constructed by weighted averaging of estimates from opposite traveling directions. Shear wave speed results for phantoms with gelatin concentrations of $5 \%, 7 \%$, and $9 \%$ were $1.52 \pm 0.10 \mathrm{~m} / \mathrm{s}, 1.86$ $\pm 0.10 \mathrm{~m} / \mathrm{s}$, and $2.37 \pm 0.15 \mathrm{~m} / \mathrm{s}$, respectively, which were consistent with estimates computed from three other conventional methods, as well as compression tests done with a commercial texture analyzer. The method was shown to be able to reconstruct a 2-D speed map of an inclusion phantom with good image quality and variance comparable to conventional methods. Suggestions for further work are given.
\end{abstract}

\section{INTRODUCTION}

$\mathrm{M}$ ECHANICAL properties of tissue can be characterized with high dynamic range by detection and quantification of shear wave velocities [1]. The equation of motion for shear wave propagation in an isotropic, homogeneous, incompressible media can be written as [2]

$$
u_{\mathrm{tt}}=c_{\mathrm{s}}^{2} \nabla^{2} u
$$

where $u$ is the 3 -D shear wave spatiotemporal signal, $u_{\mathrm{tt}}$ is the second temporal partial derivative of $u$, and $\nabla^{2} u$ is the Laplacian of $u$. For a purely elastic media, the shear modulus $\mu$ is related through the material density $\rho$ by [1]

$$
\mu=\rho\left(\frac{f_{\mathrm{mech}}}{f_{\mathrm{sp}}}\right)^{2}=\rho c_{\mathrm{s}}^{2}
$$

where $f_{\text {mech }}$ is the frequency of mechanical excitation, $f_{\mathrm{sp}}$ is the local spatial frequency, and $c_{\mathrm{S}}$ is the shear wave speed.

Imaging the elastic properties of tissue can be performed by estimating the local spatial frequency of propagation speed from a propagating shear wave. Using local

Manuscript received July 30, 2015; accepted September 14, 2015.

The authors are with the Biological Systems Engineering Department, University of Nebraska-Lincoln, Lincoln, NE 68583, USA (e-mail: gbashford2@unl.edu).

DOI http://dx.doi.org/10.1109/TUFFC.2015.007282 spatial frequency estimators, $f_{\mathrm{sp}}$ has been recovered over multiple scales and provided robust estimation of shear elasticity from a single imaging frame; however, resolution was dependent on shear wavelength and visualization of sharp boundaries was limited [3]. Phase-based estimations selectively filter for the shear wave frequency $f_{\text {mech }}$, where the phase gradient of the resulting shear wave signal provides $f_{\mathrm{sp}}$. This technique assumes propagation of a plane wave and has been implemented successfully in transient elastography [4].

Many approaches have been taken for robust estimation of shear wave speed. Inversion of (1), the secondorder wave equation, was implemented and required several imaging frames for second-order temporal derivatives [5]. By assuming independence of temporal and spatial variables, a method for inversion of the Helmholtz equation was implemented which only relied on second-order spatial derivatives. This method was able to recover shear wave speed from a single imaging frame [6]. Because the shear wave speed is estimated from the equation of motion for waves in the media, these methods assume only waves are present in the tissue. Both approaches relied on second-order derivatives which were difficult to estimate because of the inherently low SNR in the data. To overcome this limitation, time-of-flight (TOF)-based approaches to shear wave speed estimation were introduced [7]-[9]. These approaches can be broken up into various forms of cross-correlation (CC), and time-to-peak (TTP) methods. A 2-D approach for CC-based TOF speed calculation was developed and implemented to estimate shear wave speed from any direction of wave propagation [10], [11]. These approaches perform multiple cross-correlations along the direction of wave propagation to provide a moving average estimate for shear wave speed. The TTP method reduces the problem to a first-order spatial differentiation over a 2-D map representing arrival time of the maximum of the waveform.

This paper proposes a new method for 2-D shear wave speed calculation which assumes local propagation of plane waves and relies on first-order partial derivatives for a robust estimate of shear wave speed. Directional filters are used to meet the assumption of local plane shear wave propagation. This work describes the new method, including an introduction of shear waves into the media, shear wave particle velocity estimation, directional filter implementation, and post processing for final 2-D shear wave speed map estimation. The method is validated by comparing the shear modulus 1) estimated from the proposed method; 2) estimated from conventional methods; and 3) 
measured from a compression test on three homogeneous phantoms of varying Young's modulus and one inclusion phantom. The purpose of this paper is to demonstrate the feasibility of the new inversion method, and to compare and contrast the method to other current methods of shear wave speed estimation

\section{THEORY}

In a 3-D medium, the wave field $\boldsymbol{u}$ can be written as the superposition of waves originating from some source and propagating along all possible paths [12]

$$
\boldsymbol{u}(t)=\sum_{P} \boldsymbol{S}(t ; P)
$$

where $P$ is a unique path in the medium. $S$ is an arbitrary waveform associated with a particular path $P$ and may vary with time $t$ because of geometric spreading, attenuation, or interactions at interfaces along the path. By grouping terms in (3) based on the direction each path takes through space, the wave field can be rewritten as

$$
\boldsymbol{u}(t)=\sum_{\theta} \boldsymbol{U}(t ; \theta)
$$

where $\mathbf{U}$ is an arbitrary time-varying waveform propagating in the direction $\theta$ :

$$
\boldsymbol{U}(t ; \theta)=\sum_{S(P) \in \theta} \boldsymbol{S}(t ; P) .
$$

By defining $\xi$ as a new axis along the direction of wave propagation, the arbitrary waveform $\mathbf{U}$ can be defined as existing on the characteristic curve $\xi-c_{\mathrm{S}} t=0$. Assuming the shape of $\mathbf{U}$ changes only because of attenuation and not through pathway interactions, (5) becomes

$$
\begin{aligned}
\boldsymbol{U}(\xi, t) & =\boldsymbol{U}_{0}\left(\xi-c_{\mathrm{s}} t\right) e^{-\lambda t}, \quad c_{\mathrm{s}}>0, \quad \lambda \geq 0 \\
\boldsymbol{U}_{0}(\xi) & =\boldsymbol{U}(\xi, 0) .
\end{aligned}
$$

By the method of characteristics, (6) is the solution to the 1-D first-order wave equation

$$
\boldsymbol{U}_{\mathrm{t}}+c_{\mathrm{s}} \boldsymbol{U}_{\xi}=-\lambda \boldsymbol{U}, \quad c_{\mathrm{s}}>0, \quad \lambda \geq 0,
$$

where $\boldsymbol{U}_{\mathrm{t}}$ and $\boldsymbol{U}_{\xi}$ are the first derivatives of time and space, respectively. The local inversion algorithm can be written as

$$
c_{\mathrm{s}}=\left|\frac{\boldsymbol{U}_{\mathrm{t}}+\lambda \boldsymbol{U}}{\boldsymbol{U}_{\xi}}\right|, \quad \lambda \geq 0,
$$

where the absolute value forces a positive shear wave speed. Because $\boldsymbol{U}_{\mathrm{t}}=O(\omega \boldsymbol{U})$ and for weakly attenuating waves $\omega \gg \lambda$, the leading order approximation becomes

$$
c_{\mathrm{s}} \sim\left|\frac{\boldsymbol{U}_{\mathrm{t}}}{\boldsymbol{U}_{\xi}}\right| .
$$

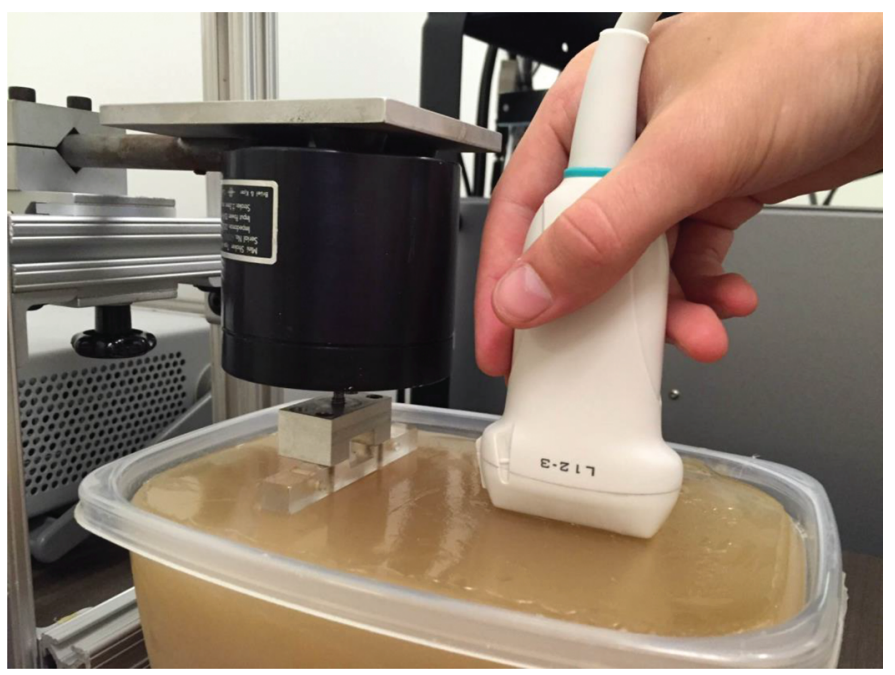

Fig. 1. Experimental setup, the L12-3v transducer is placed perpendicular to the long end of the rod attached to the shaker.

Thus, for this method, it is required that $\boldsymbol{U}$ be continuous and differentiable.

\section{Materials AND Methods}

For validation, three homogeneous gelatin phantoms were made using 5\%,7\%, and $9 \%$ gelatin by weight, and one inclusion phantom was made which was composed of $5 \%$ gelatin as a background and $9 \%$ gelatin in the inclusion. All phantoms contained $1 \%$ propanol by weight. A programmable research ultrasound system (Vantage 256, Verasonics Inc., Kirkland WA, USA) was configured to provide both the B-mode image and the shear wave motion speed map by the inversion method proposed in the preceding section. A wideband, small-parts linear-array transducer with a center frequency of $10.0 \mathrm{MHz}$ was used for imaging (L12-3v, Verasonics Inc.). Each phantom was imaged in five spatially different locations and shear wave speeds were estimated using the method presented here.

Shear waves were introduced by an external mechanical shaker (Type 4810, Brüel and Kjaer, Nærum, Denmark). A 20-cycle, $3 \mathrm{~V}$ amplitude, $200 \mathrm{~Hz}$ signal was produced by a function generator (Aglient 33250A, Agilent Technologies Inc., Santa Clara, CA, USA). A power amplifier (Type 2716c, Brüel and Kjaer) with a gain of $18 \mathrm{~dB}$ was used to drive the shaker to introduce detectable shear waves in the phantoms. To induce shear waves into the media and limit the direction of wave propagation to the dominant path (directly from the shaker), a bar was used at the phantom-shaker interface [13]. The transducer was placed perpendicular to the long axis of the bar as shown in Fig. 1.

Immediately following cessation of the external mechanical excitation, a high-frame-rate plane wave imaging method was used to reduce SNR and provide the frame rate necessary for visualization of shear wave motion [14], [15]. We used a pulse repetition frequency (PRF) of 6000 

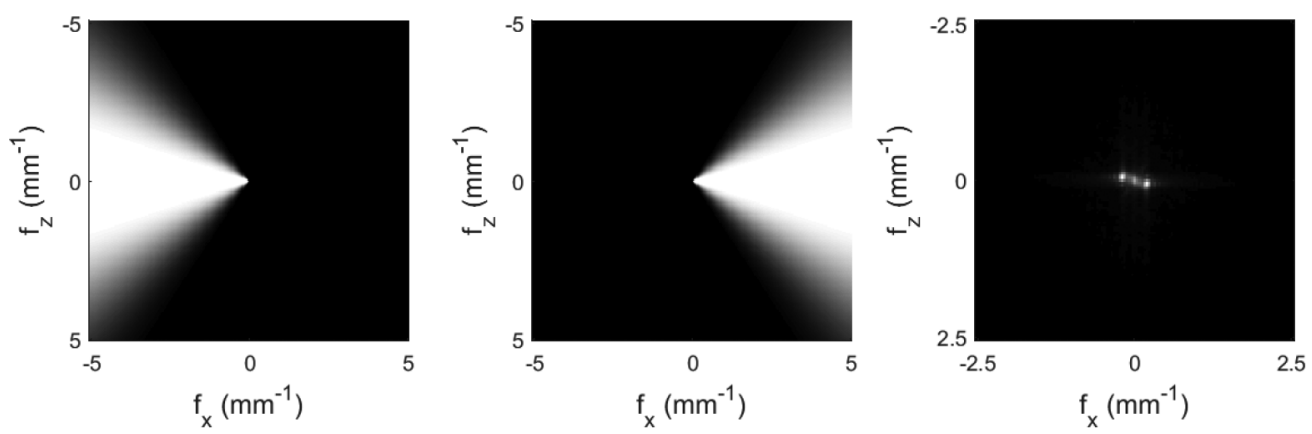

Fig. 2. Directional window and spatial filter mask used in filtering the raw shear wave signal after 1-D autocorrelation. Slices are taken from positive temporal frequencies. (left) Filter mask for isolating waves propagating right-to-left. (center) Filter mask for isolating waves propagating left-to-right. Slices are shown from the positive temporal frequencies. (right) The power spectral density of the spatial frequencies averaged over all time. The axis is magnified for more detail.

$\mathrm{Hz}$ and pulse center frequency of $10 \mathrm{MHz}$. Images were captured at three transmit angles $\left(-4^{\circ}, 0^{\circ}, 4^{\circ}\right)$. Plane wave images were formed using a pixel-based beamforming approach [16]. Axial and lateral spatial sampling was $0.1 \mathrm{~mm}$. The final 3-D in-phase/quadrature (IQ) data size was 256 $\times 256$ spatial pixels and 129 frames in time. Shear waves were imaged following cessation of external mechanical vibration to ensure the entire imaging plane contained shear waves and could be used for speed estimation. The total time for data collection was $121.5 \mathrm{~ms}$, which included 100 ms used for mechanical excitation.

Assuming no discontinuity in the shear waves, the order of partial differentiation is interchangeable, and any partial derivative of a solution is also a solution. Because the wave field is independent between dimensions, each dimension provides a solution. Thus the axial velocity component of the shear wave field is a solution to the wave equation for shear wave propagation and can be used in the inverse problem. The axial component of shear wave velocity was estimated using a two-sample 1-D autocorrelation method on the IQ data from consecutive frames of the plane wave images taken from the same angle [17]. To remove noise, a $3 \times 3$ pixel $(0.3 \mathrm{~mm} \times 0.3 \mathrm{~mm})$ spatial median filter was used on each frame in the shear wave signal. A three-sample temporal moving average of the velocity was performed to improve the SNR. A final 5 $\times 5$ pixel $(0.5 \times 0.5 \mathrm{~mm})$ spatial average was applied to the velocity estimate. The shear wave imaging frame rate remained $6000 \mathrm{~Hz}$.

Wideband directional filters similar to those used in [18] and [19] were designed to separate two directions of shear wave propagation $\left(\theta=0^{\circ}, 180^{\circ}\right)$, where the dominant path of wave propagation was assumed to be laterally across the phantom. Directional filtering was done in the frequency domain in cylindrical coordinates. Radial, azimuthal, and height coordinates correspond to spatial frequency, direction, and temporal frequency. Fig. 2 shows the combination of spatial frequency and directional windowing filters. Because waves propagating in the elevational direction may correspond to higher speeds, shear wave speeds higher than $5 \mathrm{~m} / \mathrm{s}$ were filtered out. These were filtered using a fourth-order high-pass Butterworth filter with -3 -dB cutoff frequency of $0.4 \mathrm{~mm}^{-1}$. The windowing function for the directional filtering was a Tukey window with a tapered-to-constant ratio of 0.75 [20], where the bounds of the Tukey window were at $\pm \pi / 3$ about the desired direction. Fig. 3 shows the temporal frequency filter, which was a sixth-order band-pass Butterworth filter with -3 - $\mathrm{dB}$ cutoff frequencies of $128 \mathrm{~Hz}$ and $1.38 \mathrm{kHz}$ to selectively filter the $200 \mathrm{~Hz}$ excitation signal seen in the power spectral density (PSD). Negative frequencies were kept at zero and the positive frequencies were doubled to ensure no loss in signal power.

Directional filtering was performed in the frequency domain for selection of laterally moving waves. For a robust 3-D Fourier transformation, every dimension of the spatiotemporal shear wave motion signal was Tukey windowed with a ratio of tapered-to-constant section of 0.1 . A Tukey window was chosen because it better preserves the data for shear wave speed estimation. Using an inverse 3 -D Fourier transform for each direction, the spatiotemporal-frequency shear wave signal was converted back to the spatiotemporal domain to provide the analytic shear wave signal [21].

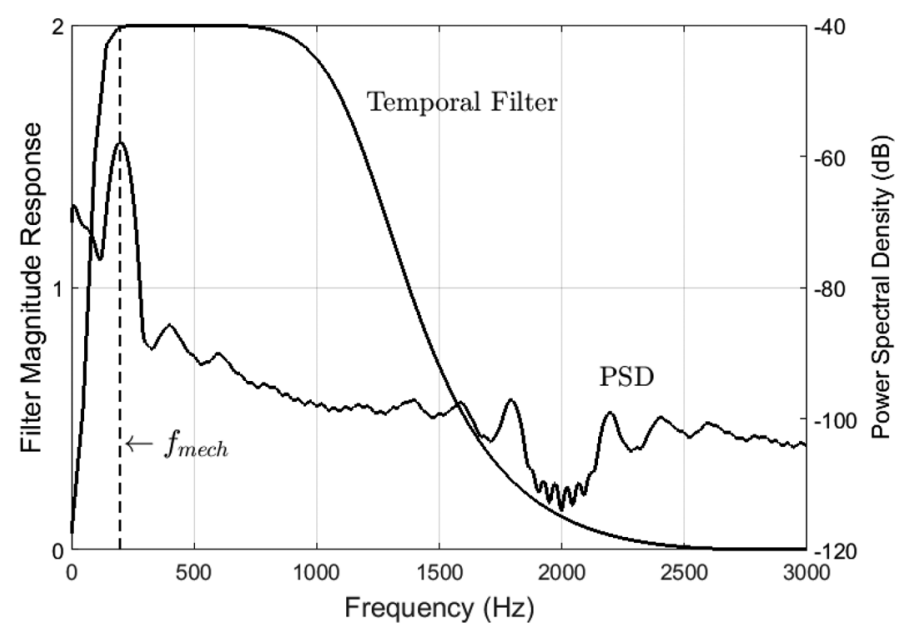

Fig. 3. Positive temporal frequency filter to isolate shear waves at the mechanical excitation frequency, negative temporal frequencies remain zero. The vertical dashed line indicates the mechanical excitation frequency of $200 \mathrm{~Hz}$ as seen in the PSD. 
Because the shear wave may propagate in the lateral and axial directions, it is necessary to estimate the $2-\mathrm{D}$ shear wave speed. Assuming the post-directionally filtered waves are unidirectional within the estimation kernel, the gradient magnitude points in the direction of wave propagation and therefore was used as an estimate for the spatial derivative, $\boldsymbol{U}_{\xi}=\sqrt{\boldsymbol{U}_{x}^{2}+\boldsymbol{U}_{z}^{2}}$. The local shear wave inversion used was

$$
c_{\mathrm{s}}=\left|\frac{\boldsymbol{U}_{\mathrm{t}}}{\sqrt{\boldsymbol{U}_{x}^{2}+\boldsymbol{U}_{z}^{2}}}\right| .
$$

Robust numerical differentiation was performed by estimating $\boldsymbol{U}_{x}$ and $\boldsymbol{U}_{z}$ using 2-D Savitzy-Golay filters with a fourth-order polynomial over a $45 \times 45$ pixel region, and $\boldsymbol{U}_{\mathrm{t}}$ was estimated using a 1-D fourth-order Savitzy-Golay filter over 21 frames in the waveform [22], [23]. The final shear wave speed map was calculated by weighted averaging of the directionally filtered speed maps. Weights were calculated in each frame from the percentage of total energy contained at each pixel in the shear wave signal for all valid frames [24]. Only frames unaffected by the temporal Tukey windowing were used for averaging. A total of 214 speed maps $(|\theta|=2,|n|=107)$ were used in the weighted averaging to produce the final shear wave speed map, given by

$$
c_{\mathrm{s}}(x, z)=\sum_{n} \sum_{\theta} c_{\mathrm{s}}(x, z, n, \theta) \frac{|\boldsymbol{U}(x, z, n, \theta)|^{2}}{\sum_{n, \theta}|\boldsymbol{U}(x, z, n, \theta)|^{2}} .
$$

No further spatial averaging was performed on the final shear wave speed estimate.

For comparison to conventional methods, the same post-directionally filtered shear wave data were used to estimate the shear wave speed. The method for 2-D CC used a spatial step size of 20 pixels $(2.0 \mathrm{~mm})$, and performed subsample peak estimation by cubic spline interpolation around the maximum point. All spatial and temporal differentiation filters used in the study were kept constant. Because speed estimation via the TTP method uses cyclical shear waves, this method requires selection of a single shear wave from the data set. For the homogeneous phantoms, a region of interest (ROI) was chosen from the center of each final shear speed map with a spatial size of $15 \times 15 \mathrm{~mm}$ and the mean and standard deviation were taken from the pixels in the ROI. The inclusion phantom contained two ROIs, $5 \times 20 \mathrm{~mm}$ for the background and
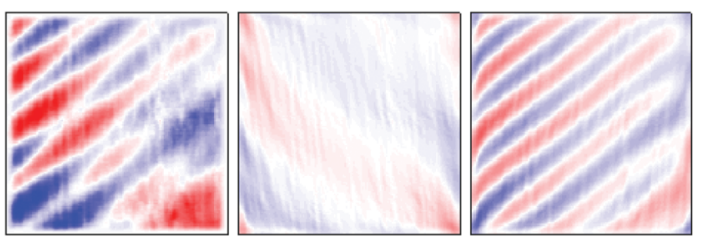

(a)
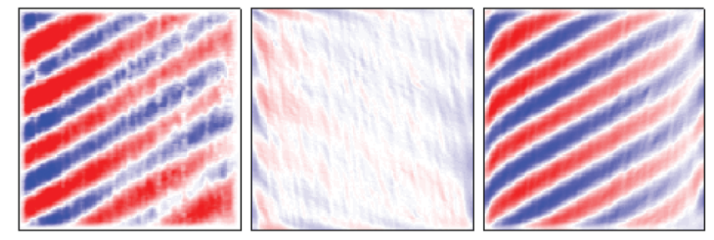

(b)
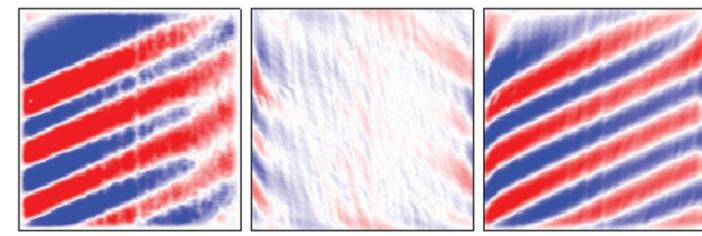

(c)
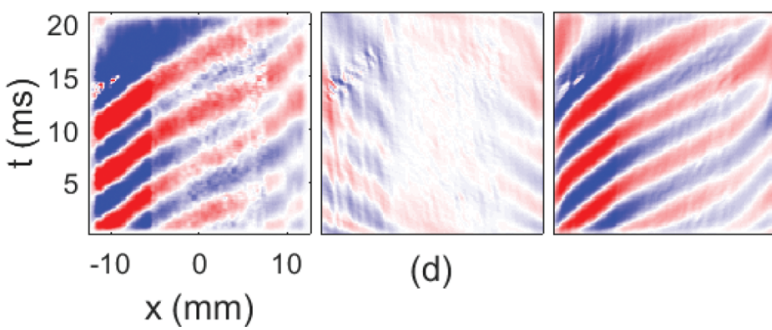

(d)

$\mathrm{mm} / \mathrm{s}$

Fig. 4. Slices in the $x t$-plane taken from a depth of $12.6 \mathrm{~mm}$ in the 3 -D spatiotemporal wave field from a (a) $5 \%$ gelatin phantom, (b) $7 \%$ gelatin phantom, (c) $9 \%$ gelatin phantom, and (d) inclusion phantom. Left, center, and right columns indicate pre-directionally filtered waves, right-toleft propagating waves, and left-to-right propagating waves, respectively. All figures use the same color bar and axis.

an $11 \mathrm{~mm}$ diameter ROI for the inclusion. Contrast-tonoise ratio was calculated by

$$
\mathrm{CNR}=\frac{\left|\bar{c}_{\mathrm{sI}}-\bar{c}_{\mathrm{sB}}\right|}{\sigma_{\mathrm{B}}},
$$

where $\bar{c}_{\mathrm{SI}}$ and $\bar{c}_{\mathrm{sB}}$ are the mean shear speed of the inclusion and background respectively and $\sigma_{\mathrm{B}}$ is the standard deviation of the background shear speed. Nominal shear wave speed was estimated from the average Young's modulus as measured using a compression test (TMS-Pro, Food Tech-

table I. Shear Wave Speed Measured From Individual Phantoms.

\begin{tabular}{llcl}
\hline & \multicolumn{3}{c}{ Shear wave speed $(\mathrm{m} / \mathrm{s})$} \\
\cline { 2 - 4 } & $5 \%$ phantom & $7 \%$ phantom & $9 \%$ phantom \\
\hline TMS-Pro & $1.56 \pm 0.05$ & $2.12 \pm 0.12$ & $2.54 \pm 0.10$ \\
Experiment & $1.52 \pm 0.10$ & $1.86 \pm 0.10$ & $2.37 \pm 0.15$ \\
Second-order wave equation & $1.45 \pm 0.07$ & $1.87 \pm 0.11$ & $2.41 \pm 0.32$ \\
2-D TTP & $1.54 \pm 0.06$ & $1.83 \pm 0.29$ & $2.36 \pm 0.16$ \\
2-D CC & $1.55 \pm 0.08$ & $2.04 \pm 0.10$ & $2.69 \pm 0.20$ \\
\hline
\end{tabular}



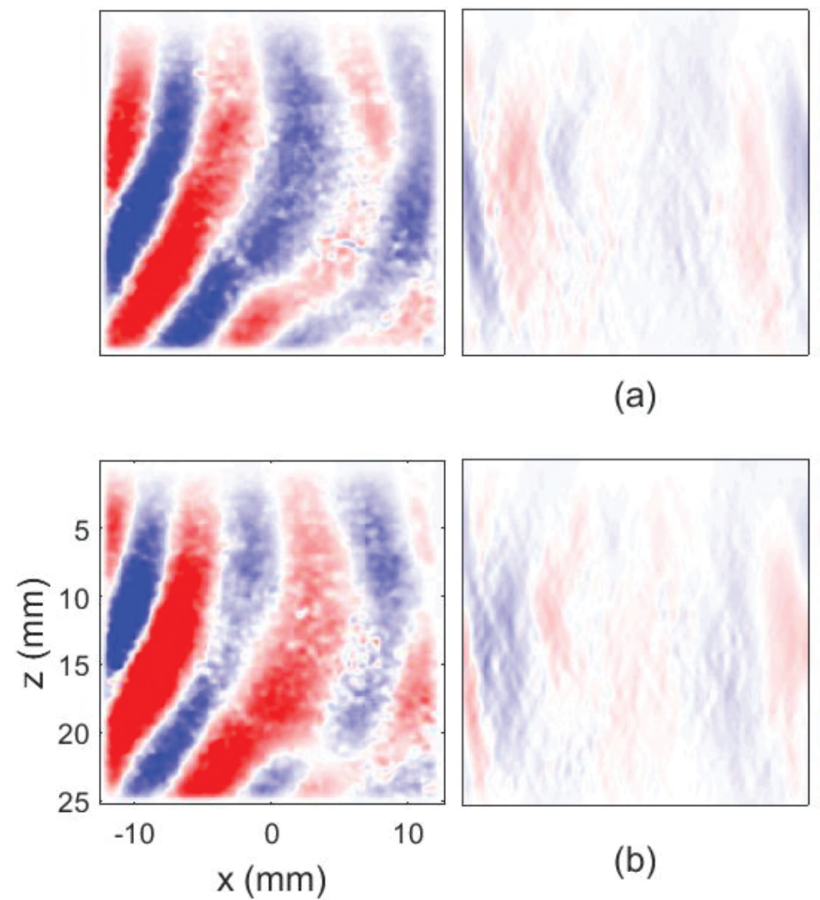

(a)

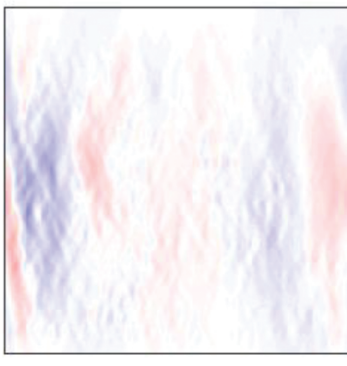

(b)
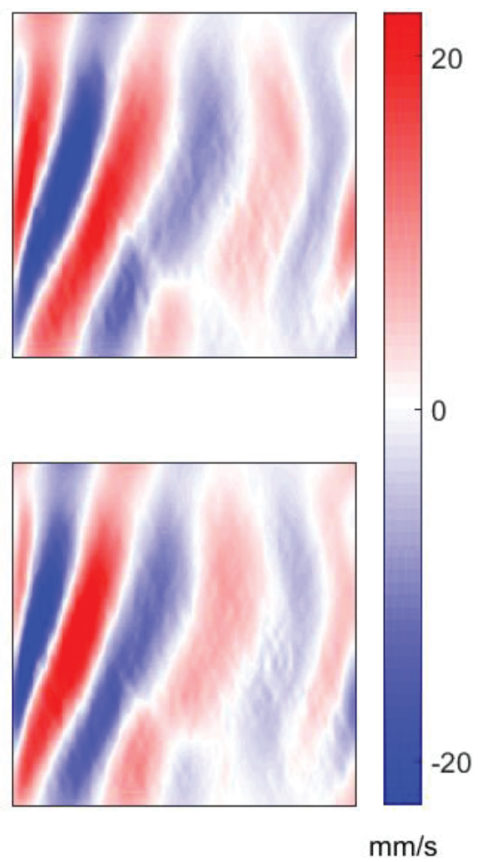

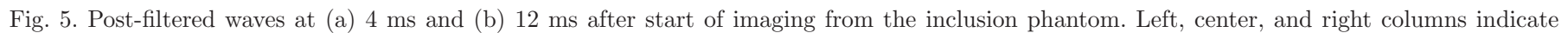

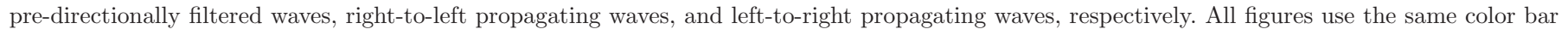
and axis.

nology Corp., Sterling, VA, USA). Compression tests consisted of five samples $\left(\sim 1 \mathrm{~cm}^{3}\right)$ taken from each phantom, and compressed at a rate of $2 \mathrm{~mm} /$ second for $1 \mathrm{~s}$. Young's modulus was calculated from the average slope of the stress-strain curve and nominal shear wave speed was calculated from the relationship $E=3 \rho c_{\mathrm{s}}^{2}$, where $\rho=1000$ $\mathrm{kg} / \mathrm{m}^{3}$ was assumed for all phantoms. Compression tests were performed within $2 \mathrm{~h}$ of the shear wave imaging.

\section{RESUlts}

Fig. 4 shows the $x$ - $t$ plane of the 3 -D spatio-temporal data taken from the center of each phantom (depth of 12.6 $\mathrm{mm}$ ). The inverse slope of the characteristic curves is representative of the shear wave speed. Fig. 5 shows representative images of the shear wave signal from the inclusion phantom taken at $4 \mathrm{~ms}$ and $12 \mathrm{~ms}$ after start of imaging. The corresponding $c_{\mathrm{S}}$ estimation is shown in Fig. 6. Similar speed maps were acquired from all other imaging frames. For energy-based weighted averaging, $c_{\mathrm{s}}$ estimates were multiplied by their energy content normalized to all energy in the post directionally filtered waves at that pixel. Fig. 7 shows the amplitude of the left-to-right propagating wave. For the inclusion phantom, the right-to-left propagating wave was negligible and is not shown. Fig. 8 shows representative estimates of $c_{\mathrm{S}}$ using the proposed method for three homogeneous phantoms. For all methods, representative means and standard deviation across the pixels within the ROI are shown in Table I. These are similar across all images taken for each homogeneous phantom. Fig. 9 shows the comparison of the average shear wave speed from the compression test, the proposed method of inversion of the first-order wave equation, inversion of the second-order wave equation, 2-D TTP, and a 2-D CC. Because the compression test provides only an average estimate of shear wave speed for each sample, the mean and
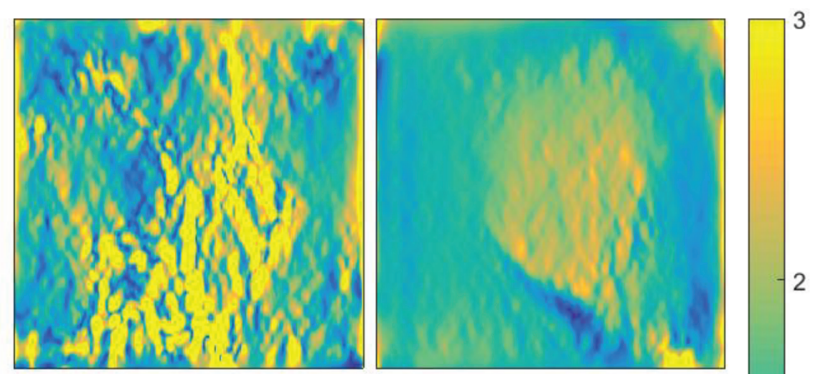

(a)
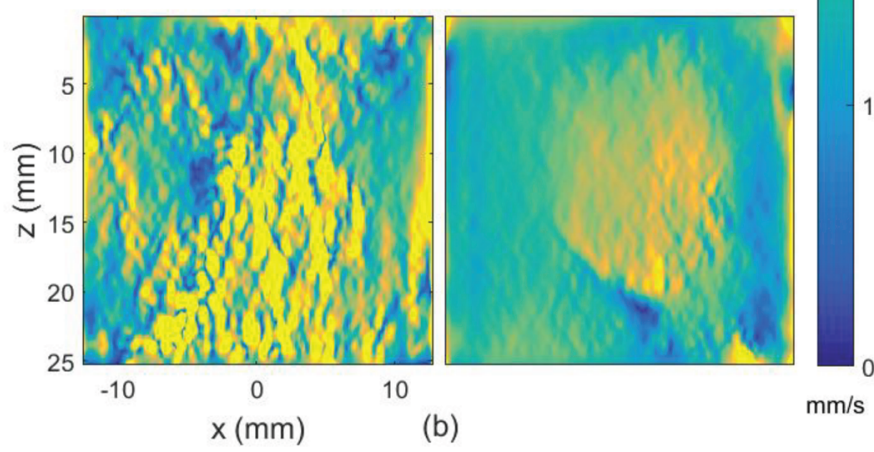

Fig. 6. Representative single frame shear wave speed estimates from the inclusion taken at a time of (a) $4 \mathrm{~ms}$ and (b) $12 \mathrm{~ms}$ after start of imaging. Left and right columns are from right-to-left and left-to-right propagating waves, respectively. All figures use the same color bar and axis. 

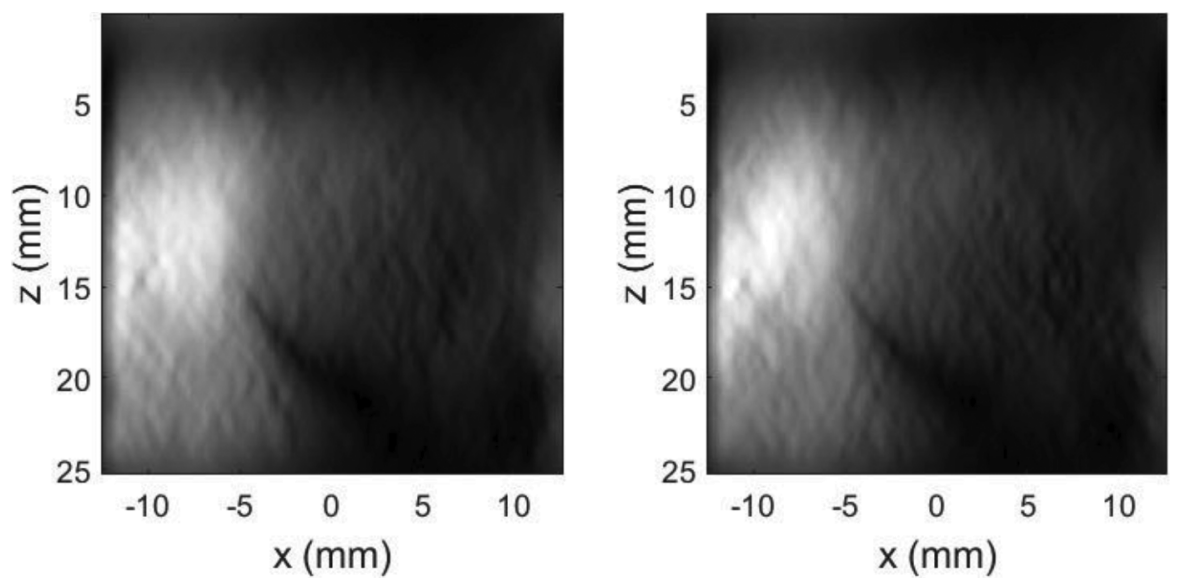

Fig. 7. Shear wave amplitude for the inclusion phantom using the left-to-right moving wave. Estimates were taken from times of (left) 4 ms, and (right) $12 \mathrm{~ms}$ after the start of imaging. After squaring and normalization through time, these amplitude estimates were used as weights for the weighted averaging of speed estimates. Energy from right-to-left moving waves is negligible and not shown.

standard deviations for the shear wave methods shown in Fig. 9 are taken from the average estimate of shear wave speed calculated from the ROI for each imaging sequence. Fig. 10 shows the inclusion phantom estimated from each shear wave speed estimation method. Table II shows the data used for CNR estimation for each of the methods, where statistics were taken from the ROIs shown in Fig. 10(a). Computational time for a single direction $(256 \times$ 256 pixel) 2-D shear wave speed map on a general computing desktop with an Intel i5 CPU (Intel Corp., Santa Clara, CA, USA) at $2.8 \mathrm{GHz}$ took $5 \mathrm{~s}$ in MATLAB (The MathWorks Inc., Natick, MA, USA) with no optimization for reduced reconstruction time.

\section{Discussion}

There are a few approaches to estimate shear wave speed from (6). The proposed method performs an inversion of the associated partial differential equation and estimates $c_{\mathrm{S}}$ from (9). Further methods include TTP and CC-based approaches. These methods assume some part of the shape of the wave does not significantly change as it propagates along the characteristic curve. TTP tracks a single point on the waveform (typically a maximum point), and estimates speed based on movement of the single chosen point, whereas the CC method relies on all points in the waveform. TTP allows for the possibility of multiple peak tracking, where errors from noise can be smoothed out as more peaks pass over a region. For CC, estimation of $c_{\mathrm{s}}$ is performed across all time and a single estimate for $c_{\mathrm{S}}$ is recovered. Because periodic shear waves were imaged, a CC-based method for $c_{\mathrm{S}}$ estimation might suffer from errors due to false peaks [25]. This may not be a problem when imaging a transient pulse; care must be taken when imaging cyclical waves, perhaps by using a priori knowledge of expected speeds. The proposed method of direct inversion of (7) relies on first-order differentiation for more robust estimates of temporal and spatial derivatives, and provides an estimate of $c_{\mathrm{s}}$ at every time sample where the wave is present. Compounding multiple $c_{\mathrm{S}}$ estimates per direction provides for a robust final estimate. Because an elasticity estimate can be made with very few imaging frames, real-time elasticity estimation may be simplified using this method. By implementing the proposed method, shear wave speed can be estimated efficiently and accurately while using conventional laboratory equipment, and with improving GPU capabilities, further improvements may be made which only require the number of frames necessary for temporal differentiation and filtering.
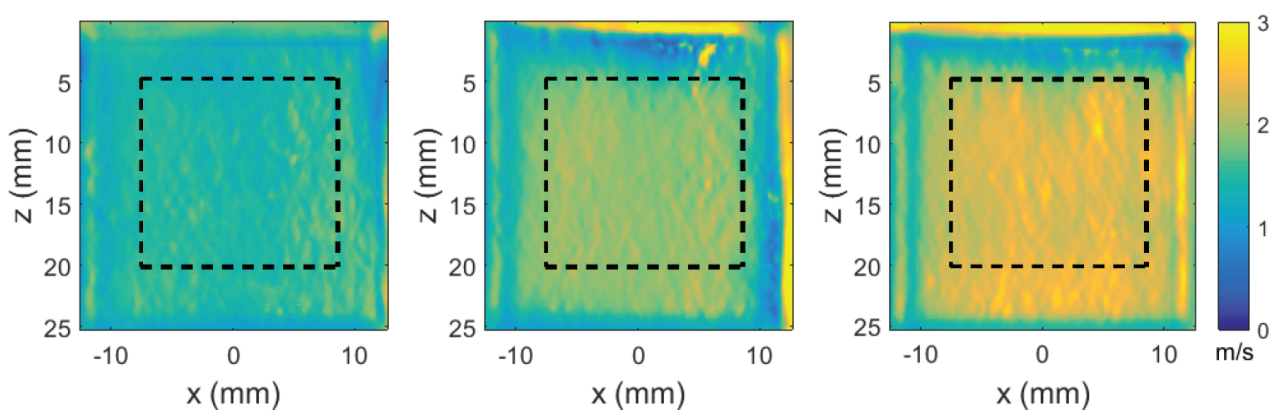

Fig. 8. Final 2-D shear speed estimates for homogeneous phantoms from the proposed 2-D shear wave speed estimation method. (left) $5 \%$ gelatin phantom $1.52 \pm 0.10 \mathrm{~m} / \mathrm{s}$, (center) $7 \%$ gelatin phantom $1.86 \pm 0.10 \mathrm{~m} / \mathrm{s}$, (right) $9 \%$ gelatin phantom $2.37 \pm 0.15 \mathrm{~m} / \mathrm{s}$. The dashed box indicates the $15 \times 15 \mathrm{~mm}$ ROI used for analysis. 
Table II. Shear Wave Speed Measured From the Inclusion Phantom.

\begin{tabular}{lrrr}
\hline & & Shear wave speed $(\mathrm{m} / \mathrm{s})$ \\
\cline { 2 - 4 } & Background & Inclusion & CNR \\
\hline Experiment & $1.50 \pm 0.06$ & $2.24 \pm 0.23$ & 12.47 \\
Second-order wave equation & $1.47 \pm 0.06$ & $2.33 \pm 0.44$ & 14.19 \\
2-D TTP & $1.50 \pm 0.07$ & $2.24 \pm 0.17$ & 10.92 \\
2-D CC & $1.58 \pm 0.07$ & $2.34 \pm 0.19$ & 11.24 \\
\hline
\end{tabular}

Estimates made on homogeneous phantoms using the proposed method are comparable to those found from inversion of the second-order wave equation, 2-D TTP, and 2-D CC methods. From Fig. 9, because each measurement was taken from an image in different spatial locations of the phantom, the variance between the mean $c_{\mathrm{s}}$ values from each image within the same phantom suggests good repeatability of measurement and homogeneity throughout the phantom. Images made on the inclusion phantom are comparable with the proposed method and most closely match the 2-D CC method. Each method estimated a slow shear wave speed directly below the inclusion. This estimation was caused by the splitting of the shear waves, which introduces a sharp gradient perpendicular to the direction of wave propagation and breaks the assumption of a plane wave in the estimation kernel. Further studies which track the true direction of wave propagation may help to decrease this phenomenon.

Shear waves were generated by a mechanical excitation from a single location; thus, shear waves were assumed to resemble plane waves propagating away from the source with little to no reflection from boundaries or inclusions. In this experiment, the shear wave is seen to propagate both laterally and axially. Because the direction of propagation may not be known entirely beforehand, to capture most of the energy in a single direction and maintain the form of the wave, wideband directional filters were implemented using a Tukey window with a tapered-to-constant ratio of 0.75 , where the window was $\pm \pi / 3$ about the desired direction of wave propagation. The truncated directional bounds allowed the Tukey window to have smooth/ sharp drop off while maintaining directionality. If shear waves were assumed to be propagating in unknown directions, or generated at several angles by acoustic radiation force, several narrower directional filters would need to be implemented. This would increase SNR and provide a smoother final image, but may filter out sharp boundaries between tissues of significantly different shear wave speed. Regardless of the number of directional filters, the method proposed here assumes the shear wave is approximated by a plane wave in the estimation kernel. A minimum number of directional filters should be used to meet this assumption. A study with different directional windowing functions might better describe reconstruction image quality for final $c_{\mathrm{s}}$ estimates.

Energy-based compounding was used because higher shear wave energy increases the SNR used for shear wave speed estimation. In homogeneous media, the shear wave can be assumed to occupy the entire imaging frame. For complex media such as a tendon, several layers of differing shear modulus may be present in the imaging frame and shear waves with high SNR may only exist in a given layer for a fraction of the imaging time. With overlapping waves propagating in multiple directions, attenuation due to viscosity lowers the energy of the waves over time and estimates closer to the source contain a higher SNR. Strong reflection from boundaries within the media may also be used for estimates. The energy-based compounding method implemented here allows for selective weighted averaging at times where the initial shear waves are no longer present or where reflected waves may be more dominant.

This study used 20 cycles of external mechanical excitation for generation of near steady-state conditions for propagation of shear waves over the entire media. This can be seen in Fig. 4, where the post-directionally filtered waves cover the entire lateral imaging region for the duration of imaging. Although the external shaker requirement may reduce portability and practicality in most clinical applications, it provides more force for generation of shear waves for imaging elasticity deep with the media or in much stiffer tissue. Thus, development of an unobtrusive fixation device for an external excitation source may improve usability while providing the force needed to image stiff (such as tendon) or deep tissues.

The use of acoustic-radiation-force-based SWE allows for the control of shear wave excitation and limits the

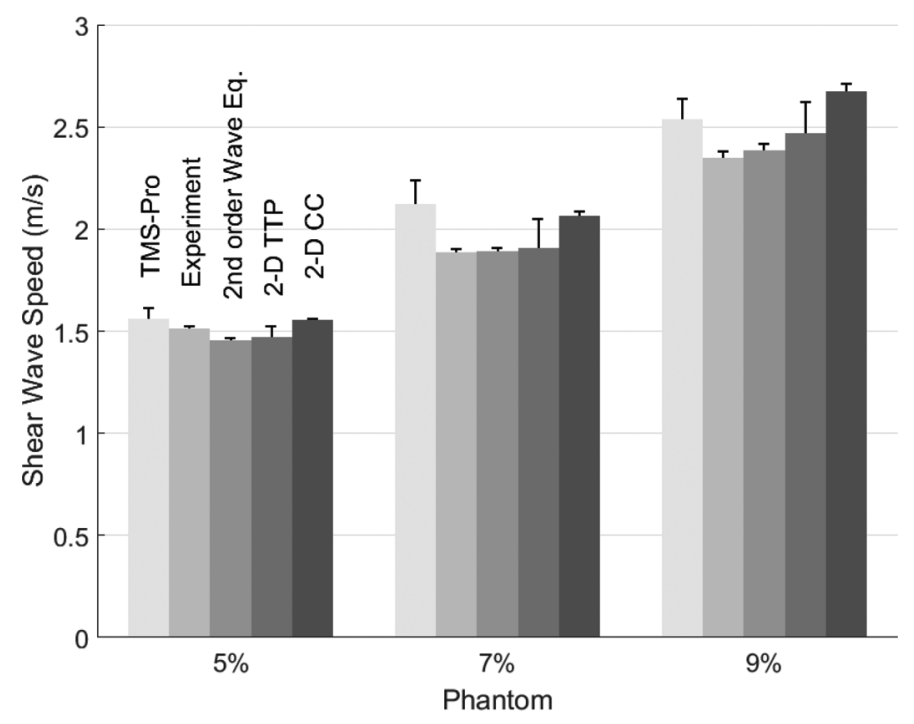

Fig. 9. Shear wave speed estimates from the proposed experimental method and the compression test for phantoms of $5 \%, 7 \%$, and $9 \%$ gelatin concentrations. 


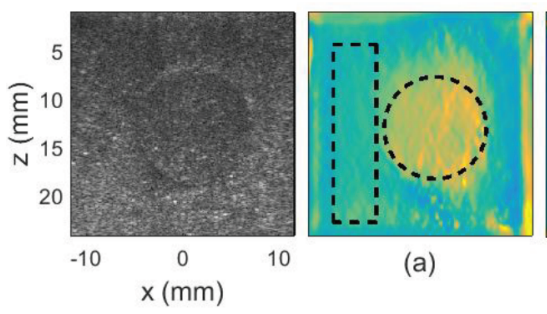

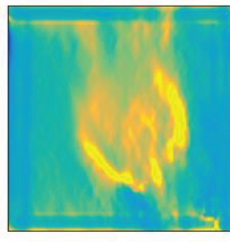

(b)

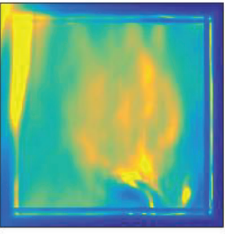

(c)

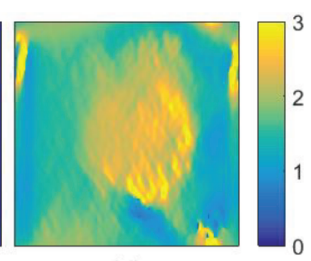

(d)

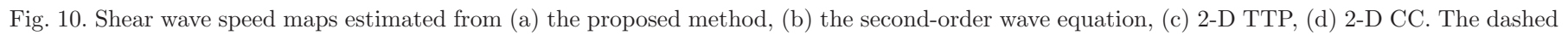

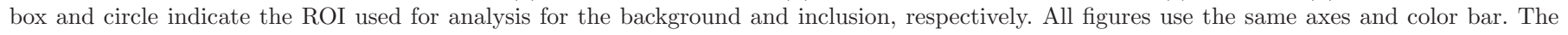
corresponding B-mode image is shown on the left for reference.

dominant path of wave propagation in the imaging plane. Because imaging and shear wave excitation are coupled, this may provide improved system usability and reduce shear speed estimation variance over multiple imaging acquisitions. However, because the shear wave only resides in portions of the image at one time, the lower total shear wave energy may reduce the SNR and degrade the estimate for shear speed. This may be overcome by introducing multiple acoustic impulses in a single acquisition event [26]. A study implementing the proposed method of $c_{\mathrm{S}}$ estimation on transient waves produced from acoustic radiation might better show its use in acoustic-radiationforce-based SWE.

\section{Conclusion}

This study presented a new inversion method using first-order differentiation for robust estimation of 2-D shear wave propagation speed. This method was validated on homogeneous and inclusion phantoms and results were comparable to measurements from a compression test and three other conventional shear wave speed estimation methods. Implementation on a dedicated device with optimized hardware and software should allow for efficient real time imaging of the elastic modulus of tissue. Possible future work would include studying the performance of this method in more complex media such as in vivo tissues.

\section{ACKNOWLEDGMENTS}

The authors thank the Nebraska Athletics Performance Laboratory for use of their Verasonics System.

\section{REFERENCES}

[1] A. P. Sarvazyan, M. W. Urban, and J. F. Greenleaf, "Acoustic waves in medical imaging and diagnostics," Ultrasound Med. Biol., vol. 39, no. 7, pp. 1133-1146, Jul. 2013.

[2] L. Sandrin, M. Tanter, S. Catheline, and M. Fink, "Shear modulus imaging with 2-D transient elastography," IEEE Trans. Ultrason. Ferroelectr. Freq. Control, vol. 49, no. 4, pp. 426-435, Apr. 2002.

[3] A. Manduca, T. E. Oliphant, M. a. Dresner, J. L. Mahowald, S. a. Kruse, E. Amromin, J. P. Felmlee, J. F. Greenleaf, and R. L. Ehman, "Magnetic resonance elastography: Non-invasive mapping of tissue elasticity," Med. Image Anal., vol. 5, no. 4, pp. 237-254, Dec. 2001.

[4] S. Catheline, F. Wu, and M. Fink, "A solution to diffraction biases in sonoelasticity: The acoustic impulse technique," J. Acoust. Soc. Am., vol. 105, no. 5, pp. 2941-2950, 1999.

[5] J. Bercoff, M. Tanter, and M. Fink, "Supersonic shear imaging: A new technique for soft tissue elasticity mapping," IEEE Trans. Ultrason. Ferroelectr. Freq. Control, vol. 51, no. 4, pp. 396-409, 2004.

[6] T. E. Oliphant, A. Manduca, R. L. Ehman, and J. F. Greenleaf, "Complex-valued stiffness reconstruction for magnetic resonance elastography by algebraic inversion of the differential equation," Magn. Reson. Med., vol. 310, no. 2, pp. 299-310, 2001.

[7] J. McLaughlin and D. Renzi, "Shear wave speed recovery in transient elastography and supersonic imaging using propagating fronts," Inverse Probl., vol. 22, no. 2, pp. 681-706, Apr. 2006.

[8] M. L. Palmeri, M. H. Wang, J. J. Dahl, K. D. Frinkley, and K. R. Nightingale, "Quantifying hepatic shear modulus in vivo using acoustic radiation force," Ultrasound Med. Biol., vol. 34, no. 4, pp. 546-558, 2008.

[9] M. Tanter, J. Bercoff, A. Athanasiou, T. Deffieux, J.-L. Gennisson, G. Montaldo, M. Muller, A. Tardivon, and M. Fink, "Quantitative assessment of breast lesion viscoelasticity: Initial clinical results using supersonic shear imaging," Ultrasound Med. Biol., vol. 34, no. 9, pp. 1373-1386, Sep. 2008.

[10] P. Song, A. Manduca, H. Zhao, M. W. Urban, J. F. Greenleaf, and S. Chen, "Fast shear compounding using robust 2-D shear wave speed calculation and multi-directional filtering," Ultrasound Med. Biol., vol. 40, no. 6, pp. 1343-1355, Jul. 2014.

[11] H. Zhao, P. Song, D. D. Meixner, R. R. Kinnick, M. R. Callstrom, W. Sanchez, M. W. Urban, A. Manduca, J. F. Greenleaf, and S. Chen, "External vibration multi-directional ultrasound shearwave elastography (EVMUSE): Application in liver fibrosis staging," IEEE Trans. Med. Imaging, vol. 33, no. 11, pp. 2140-2148, 2014.

[12] R. Snieder, "Imaging and averaging in complex media," in Diffuse Waves in Complex Media, J.-P. Fouque, Ed. Dordrecht, The Netherlands: Kluwer, 1999, pp. 405-454.

[13] J.-L. Gennisson, S. Catheline, S. Chaffaï, and M. Fink, "Transient elastography in anisotropic medium: Application to the measurement of slow and fast shear wave speeds in muscles," J. Acoust. Soc. Am., vol. 114, no. 1, pp. 536-541, 2003.

[14] M. Tanter, J. Bercoff, L. Sandrin, and M. Fink, "Ultrafast compound imaging for 2-D motion vector estimation: Application to transient elastography," IEEE Trans. Ultrason. Ferroelectr. Freq. Control, vol. 49, no. 10, pp. 1363-1374, Oct. 2002.

[15] M. Tanter and M. Fink, "Ultrafast imaging in biomedical ultrasound," IEEE Trans. Ultrason. Ferroelectr. Freq. Control, vol. 61, no. 1, pp. 102-119, Jan. 2014.

[16] R. E. Daigle, "Ultrasound imaging system with pixel oriented processing," US Patent 20090112095, Apr. 30, 2009.

[17] C. Kasai, K. Namekawa, A. Koyano, and R. Omoto, "Real-time twodimensional blood flow imaging using an autocorrelation technique," IEEE Trans. Sonics Ultrason., vol. 32, no. 3, pp. 458-464, 1985.

[18] A. Manduca, D. S. Lake, S. A. Kruse, and R. L. Ehman, "Spatiotemporal directional filtering for improved inversion of MR elastography images," Med. Image Anal., vol. 7, no. 4, pp. 465-473, 2003.

[19] T. Deffieux, J. Gennisson, J. Bercoff, and M. Tanter, "On the effects of reflected waves in transient shear wave elastography," IEEE Trans. Ultrason. Ferroelectr. Freq. Control, vol. 58, no. 10, pp. 20322035, 2011. 
[20] F. J. Harris, "On the use of windows for harmonic analysis with the discrete Fourier transform," Proc. IEEE, vol. 66, no. 1, pp. 51-83, 1978.

[21] S. L. Marple, "Computing the discrete-time "analytic" signal via FFT," IEEE Trans. Signal Process., vol. 47, no. 9, pp. 2600-2603, Sep. 1999.

[22] A. Savitzky and M. J. E. Golay, "Smoothing and differentiation of data by simplified least squares procedures," Anal. Chem., vol. 36, no. 8, pp. $1627-1639,1964$.

[23] J. Krumm, (2001, Aug.). Savitzky-Golay Filters for 2D Images. [Online]. Available: Microsoft Research, Microsoft Corporation, Redmond, WA 98052 http://www.research.microsoft.com/users/ jckrumm/SavGol/SavGol.htm

[24] T. Deffieux, J.-L. Gennisson, B. Larrat, M. Fink, and M. Tanter, "The variance of quantitative estimates in shear wave imaging: Theory and experiments," IEEE Trans. Ultrason. Ferroelectr. Freq. Control, vol. 59, no. 11, pp. 2390-2410, Nov. 2012.

[25] B. Ramamurthy and G. Trahey, "Potential and limitations of angleindependent flow detection algorithms using radio-frequency and detected echo signals," Ultrason. Imaging, vol. 13, no. 3, pp. 252 268, Jul. 1991.

[26] P. Song, H. Zhao, A. Manduca, M. W. Urban, J. F. Greenleaf, and S. Chen, "Comb-push ultrasound shear elastography (CUSE): A novel method for two-dimensional shear elasticity imaging of soft tissues," IEEE Trans. Med. Imaging, vol. 31, no. 9, pp. 1821-1832, 2012 .

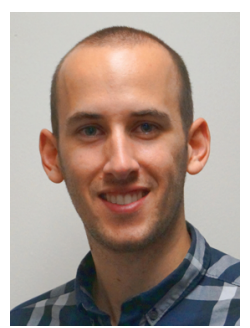

Aaron Engel was born in Phoenix, AZ, in August 1989. He received the B.S. degree in electrical engineering from the University of Nebraska-Lincoln (UNL) in 2012. He is currently a Ph.D. student in biomedical engineering at UNL. His research interests include ultrasound-based elasticity imaging methods and their applications for the detection of disease and patient management.

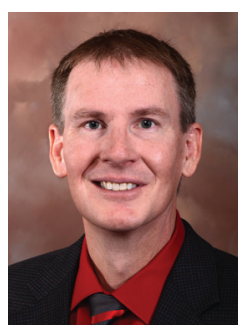

Gregory R. Bashford (M'96-SM'03) received the B.S. degree in electrical engineering from the University of Nebraska, Lincoln, NE, and the Ph.D. degree in biomedical engineering from Duke University, Durham, NC. He was previously an Image Analysis Engineer at Acuson Corporation, Mountain View, CA; Systems Engineer at GE Medical Systems, Milwaukee, WI; and Senior Scientist at LI-COR Biosciences, Lincoln, NE. In 2003, he joined the faculty of the Biological Systems Engineering Department at the University of Nebraska. His research interests include methods and applications of blood flow measurement, especially transcranial Doppler for neurological protection, and musculoskeletal health assessment using ultrasound. 